\title{
Alternativas metodológicas do teste de envelhecimento acelerado em sementes de coentro
}

\author{
Methodological alternatives for the accelerated aging test in coriander seeds
}

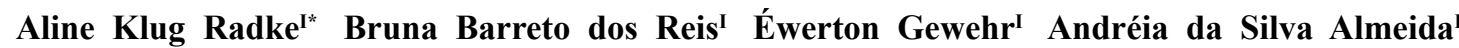 \\ Lilian Madruga de Tunes ${ }^{I}$ Francisco Amaral Villela ${ }^{I}$
}

RESUMO

No presente trabalho, o objetivo foi adequar a metodologia do teste de envelhecimento acelerado para avaliação do potencial fisiológico de sementes de Coriandrum sativum L. Seis lotes de sementes foram utilizados, sendo submetidos aos testes de germinação, indice de velocidade de emergência, emergência de plântulas e envelhecimento acelerado, empregando-se os periodos de envelhecimento de 24, 48, 72 e 96 horas, na metodologia tradicional com água e com o uso de solução saturada e não saturada de cloreto de sódio ( $\mathrm{NaCl}$ ). O teste de envelhecimento acelerado, utilizando solução salina saturada na combinação 24 horas a $41^{\circ} \mathrm{C}$ é adequado para avaliação do potencial fisiológico de sementes de coentro. O periodo de 48 horas para o método de envelhecimento acelerado, utilizando solução salina saturada ou não, também é eficaz na avaliação do vigor em sementes de coentro.

Palavras-chave: Coriandrum sativum L., hortaliça, potencial fisiológico, solução salina, vigor:

\section{ABSTRACT}

This study aimed to adapt the methodology of the accelerated aging test to assess the physiological seed of Coriandrum sativum L. Six batches of seeds were tested for germination, emergence rate index, seedling emergence and accelerated aging employing the aging periods of 24, 48, 72 and 96 hours, the traditional methodology with water and the use of unsaturated and saturated sodium chloride ( $\mathrm{NaCl}$ ) solutions. Accelerated aging test using saturated saline 24 hours in a combination with $41^{\circ} \mathrm{C}$, is adequate to evaluate the physiological potential of coriander seeds. The period of 48 hour for accelerated aging method using saturated saline or not, is also effective in the evaluation vigor test of coriander seeds.

Key words: Coriandrum sativum L., physiological potential, saline solution, vegetable, vigor.

\section{INTRODUÇÃO}

O coentro (Coriandrum sativum L.) é uma hortaliça folhosa muito utilizada na culinária brasileira, em especial na região Nordeste do Brasil, sendo uma cultura de grande rotatividade comercial. A qualidade das sementes utilizadas pelos agricultores está relacionada de forma direta ao seu potencial fisiológico, representado pela germinação e vigor, expressando sua capacidade de originar plântulas normais (PEREIRA et al., 2011).

Para determinar o potencial germinativo de um lote de sementes, utiliza-se o teste de germinação, porém, esse teste, isoladamente, não determina a efetiva qualidade da semente, dessa forma, os testes de vigor fornecem informações complementares, que permitem detectar diferenças fisiológicas para lotes de mesma porcentagem de germinação. Dentre os testes utilizados e também considerados como um dos mais sensíveis para a avaliação do vigor de sementes destaca-se o teste de envelhecimento acelerado (MARCOS FILHO, 1999), uma vez que seus resultados se relacionam com o potencial de conservação das sementes.

Um aspecto de grande relevância a ser considerado no teste de envelhecimento acelerado é o tamanho da semente, pois, segundo RAMOS et al. (2004), para espécies de sementes relativamente pequenas, como as de hortaliças, tem-se obtido resultados menos consistentes, devido à variação muito acentuada no grau de umidade ao final do

\footnotetext{
IPrograma de Pós-graduação em Ciência e Tecnologia de Sementes, Universidade Federal de Pelotas (UFPel), 96001-970, Capão do Leão, RS, Brasil. E-mail: alinekradke@hotmail.com. "Autor para correspondência.
} 
período de envelhecimento. Sementes menores absorvem água de forma rápida e desuniforme, em relação às sementes maiores, durante o período de envelhecimento, acarretando aceleração do processo de deterioração ou variação de dados entre as sementes de uma mesma amostra, interferindo na precisão dos resultados (JIANHUA \& MCDONALD, 1996; BHÉRING et al., 2006).

Nessa perspectiva, vêm sendo estudadas alternativas para a condução do teste de envelhecimento acelerado com sementes dessas espécies, como a substituição da água por soluções salinas. De acordo com a solução utilizada, podem ser obtidas umidades específicas, propiciando redução da intensidade e da taxa de absorção de água pelas sementes, culminando numa menor intensidade de deterioração e menor variação entre os resultados (JIANHUA \& MCDONALD, 1996; TUNES et al., 2012).

O emprego de solução salina saturada ou não saturada limita a absorção de água pelas sementes e o desenvolvimento de fungos, fato que pode ser atribuído à formação de atmosfera sobreposta à solução salina não propícia à proliferação de fungos (ÁVILA et al., 2006).

Diante do exposto, no presente trabalho, objetivou-se adequar a metodologia do teste de envelhecimento acelerado para avaliação do potencial fisiológico de sementes de coentro.

\section{MATERIAL E MÉTODOS}

O experimento foi conduzido no Laboratório Didático de Análise de Sementes "Flávio Rocha" da Faculdade de Agronomia Eliseu Maciel, da Universidade Federal de Pelotas (UFPel). Utilizaramse seis lotes de sementes de coentro (Coriandrum sativum L.) cultivar 'Verdão'.

A qualidade inicial das sementes foi determinada pelas seguintes avaliações: Teor de água: utilizou-se o método da estufa a $105 \pm 3^{\circ} \mathrm{C}$, por 24 horas, de acordo com as Regras para Análise de Sementes RAS (BRASIL, 2009). Foram utilizadas duas subamostras com aproximadamente $4,0 \mathrm{~g}$ de sementes para cada lote, e os resultados foram expressos em percentagem média (base úmida) por lote.

Germinação: foram utilizadas com quatro subamostras de 50 sementes por lote, distribuídas sobre duas folhas de papel mata-borrão, previamente umedecidas com quantidade de água equivalente a 2,5 vezes a massa do papel seco, e colocadas no interior de caixas de acrílico transparentes $(11 \times 11 \times 3,5 \mathrm{~cm})$, a $20^{\circ} \mathrm{C}$. As avaliações foram realizadas aos 7 e 21 dias após a semeadura e os resultados expressos em percentagem média de plântulas normais para cada lote (BRASIL, 2009).
Emergência em casa de vegetação - foram utilizadas quatro subamostras de 50 sementes por lote, distribuídas em bandejas de poliestireno expandido com células individuais, preenchidas com substrato comercial (Plantmax). As contagens foram efetuadas diariamente até a estabilização da emergência (18o dia após a semeadura), computando-se as plântulas normais emersas (tamanho igual ou superior a $1,0 \mathrm{~cm}$ ), determinando-se, então, a porcentagem de plântulas emersas (NAKAGAWA, 1999).

Índice de velocidade de emergência em casa de vegetação - realizado em conjunto com o teste de emergência de plântulas em casa de vegetação pela contagem diária do número de plântulas normais emersas (tamanho igual ou superior a $1,0 \mathrm{~cm}$ ), até a estabilização da emergência (18o dia após a semeadura). Para cada subamostra, foi calculado o índice de velocidade de emergência, somando-se o número de plântulas emersas a cada dia, dividido pelo respectivo número de dias transcorridos a partir da semeadura, conforme MAGUIRE (1962).

Após a avaliação da qualidade inicial, os lotes foram submetidos ao teste de envelhecimento tradicional e modificados, conforme descrito a seguir:

Envelhecimento acelerado (procedimento tradicional) - conduzido com a utilização de caixas de acrílico tipo gerbox, com compartimento individual (minicâmaras), contendo $40 \mathrm{~mL}$ de água, uma bandeja de tela de alumínio, onde as amostras de 4,0g de sementes foram distribuídas formando uma camada uniforme. As caixas foram mantidas em incubadora por quatro períodos de envelhecimento a $41^{\circ} \mathrm{C}$. Decorrido cada período de envelhecimento, 16 subamostras de 50 sementes por lote foram submetidas ao teste de germinação, seguindo metodologia descrita anteriormente, com avaliação realizada no sétimo dia após a semeadura, computando-se a porcentagem de plântulas normais.

Envelhecimento acelerado (solução salina saturada SSS) - conduzido como descrito para o teste de envelhecimento acelerado tradicional, porém, foram colocados, no interior de cada gerbox, $40 \mathrm{~mL}$ de solução saturada de cloreto de sódio $(40 \mathrm{~g}$ de $\mathrm{NaCl}$ em $100 \mathrm{~mL}$ de água), visando proporcionar ambiente com $76 \%$ de umidade relativa do ar, de acordo com procedimento proposto por MARCOS FILHO (2005).

Envelhecimento acelerado (solução salina não saturada SSNS) - conduzido utilizando metodologia similar ao envelhecimento acelerado com solução salina saturada, com exceção para concentração da solução $(11 \mathrm{~g}$ de $\mathrm{NaCl}$ em $100 \mathrm{~mL}$ de água), estabelecendo um ambiente com umidade relativa de $94 \%$, conforme metodologia descrita por ÁVILA et al. (2006). 
Nas três metodologias do teste de envelhecimento acelerado, empregaram-se períodos de envelhecimento de 24, 48, 72 e 96 horas, e, paralelamente aos testes de envelhecimento acelerado, foi determinado o teor de água das sementes após cada período de envelhecimento, para verificar a uniformidade das condições do teste, conforme MARCOS FILHO (1999).

Os dados em percentagem foram submetidos à transformação arc sen $\sqrt{x} / 100$ antes da análise, o delineamento experimental foi inteiramente casualizado, sendo as médias comparadas pelo teste de Tukey em nível de 5\% de probabilidade.

\section{RESULTADOS E DISCUSSÃO}

Os teores de água inicial das sementes foram semelhantes para os seis lotes (Tabela 1). O que é importante para a execução dos testes, considerando-se que a uniformização do teor de água das sementes é imprescindível para a padronização dos procedimentos e obtenção de resultados consistentes (MARCOS FILHO, 2005), uma vez que, dentro de certos limites, as sementes mais úmidas são mais afetadas pelas condições do envelhecimento acelerado (TUNES et al., 2012).

Para os resultados do teste de germinação (Tabela 1), não houve diferença entre os lotes analisados, cuja germinação manteve-se entre 80 e $88 \%$, porque é importante e coerente a comparação de lotes de sementes com germinação semelhante (MARCOS FILHO, 1999).

Tabela 1 - Teor de água (TA), germinação (G), emergência de plântulas (EP) e índice de velocidade de emergência (IVE) de lotes de sementes de coentro (Water content of coriander, germination, seedling emergence and emergence speed index coriander seed lots). Capão do Leão, RS. 2013.

\begin{tabular}{lcccl}
\hline LOTES & TA $(\%)$ & G $(\%)$ & EP $(\%)$ & IVE \\
\hline 1 & 7,0 & $82 \mathrm{a}$ & $56 \mathrm{~b}$ & $8,5 \mathrm{~b}$ \\
2 & 6,4 & $85 \mathrm{a}$ & $77 \mathrm{a}$ & $10,4 \mathrm{a}$ \\
3 & 6,6 & $81 \mathrm{a}$ & $76 \mathrm{a}$ & $11,6 \mathrm{a}$ \\
4 & 6,8 & $85 \mathrm{a}$ & $35 \mathrm{c}$ & $7,1 \mathrm{c}$ \\
5 & 6,5 & $80 \mathrm{a}$ & $60 \mathrm{~b}$ & $8,6 \mathrm{~b}$ \\
6 & 7,0 & $88 \mathrm{a}$ & $80 \mathrm{a}$ & $11,8 \mathrm{a}$ \\
(DMS) & & 8,2 & 9,6 & 1,4 \\
CV (\%) & & 9,01 & 5,64 & 7,18 \\
\hline
\end{tabular}

*Médias seguidas de mesma letra na coluna não diferem pelo teste de Tukey, em nível de probabilidade de 5\%.
No teste de emergência de plântulas (Tabela 1), observou-se diferença entre os lotes de sementes, sendo os lotes 2, 3 e 6 de vigor superior, os lotes 1 e 5 de vigor intermediário e o lote 4 de vigor inferior. Os resultados do teste de índice de velocidade de emergência em casa de vegetação possibilitaram a classificação dos lotes de forma semelhante ao observado no teste de emergência. Tanto o teste de emergência como índice de velocidade de emergência permitiram a classificação dos lotes quanto ao potencial fisiológico, não evidenciados pelo teste de germinação.

As sementes submetidas ao teste de envelhecimento acelerado empregando o procedimento tradicional (Tabela 2) atingiram maior teor de água do que aquelas submetidas aos procedimentos modificados, verificando-se a mesma tendência observada em sementes de brássicas (COSTA et al., 2008). Segundo COSTA et al. (2008), as sementes tendem a alcançar o equilíbrio higroscópico em teores de água mais elevados, conforme aumenta a umidade relativa do ar.

O teor de água corresponde ao ponto de equilíbrio, o qual aumenta com a elevação da umidade relativa do ar e vice-versa MARCOS FILHO (2005). Assim, sementes em contato com ar, cuja umidade relativa era de $100 \%$, terão maior teor de água do que sementes em contato com ar apresentando 94 e $76 \%$ de umidade relativa, respectivamente.

Os resultados demonstraram que o uso de solução não saturada assim como a saturada de $\mathrm{NaCl}$ proporcionaram menor absorção de água pelas sementes, resultados semelhantes foram encontrados por ÁVILA et al. (2006) em sementes de rabanete; por COSTA et al. (2008) em sementes de couve, couve-brócolis e repolho; por TORRES \& BEZERRA (2009) em sementes de urucum e por TUNES et al. (2013) em sementes de salsa.

$\mathrm{O}$ uso de soluções com $\mathrm{NaCl}$ tem a vantagem de proporcionar menor variação do teor de água entre as sementes e a inibição do crescimento e desenvolvimento de fungos (RODO et al., 2000), facilitando o manuseio das sementes e eliminando sua possível interferência na avaliação do potencial fisiológico das sementes durante os testes, o que foi observado em trabalhos anteriores com sementes de rabanete (ÁVILA et al., 2006); brássicas (COSTA et al., 2008); melão (TORRES et al., 2009); e coentro (PEREIRA et al., 2011).

Empregando-se96horas de envelhecimento acelerado, as variações entre os lotes atingiram 14,6 pontos percentuais em relação ao método tradicional, 6,3 pontos percentuais no método SSNS e 5,5 pontos percentuais no método SSS. 
Tabela 2 - Teor de água de lotes de sementes de coentro após quatro períodos de exposição ao teste de envelhecimento acelerado (EA), método tradicional, solução salina não saturada (SSNS), solução salina saturada (SSS). (Water content of coriander seeds after four exposure periods to the conventional accelerated aging, unsaturated salt solution and saturated salt solution tests). Capão do Leão, RS. 2013.

\begin{tabular}{|c|c|c|c|c|c|c|c|c|c|c|c|c|}
\hline \multirow[t]{2}{*}{ Lotes } & \multicolumn{4}{|c|}{------Tradicional Tempo (horas)-------- } & \multicolumn{4}{|c|}{-----------SSNS Tempo (horas)----------- } & \multicolumn{4}{|c|}{ 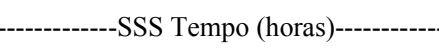 } \\
\hline & 24 & 48 & 72 & 96 & 24 & 48 & 72 & 96 & 24 & 48 & 72 & 96 \\
\hline 1 & 9,8 & 14,8 & 26,2 & 42,2 & 8,2 & 10,2 & 13,3 & 17,5 & 9,1 & 12,2 & 15,2 & 15,2 \\
\hline 2 & 8,5 & 12,5 & 24,3 & 35,3 & 7,1 & 8,5 & 9,1 & 13,6 & 7,1 & 9,8 & 10,9 & 10,8 \\
\hline 3 & 8,1 & 12,0 & 23,9 & 33,9 & 6,9 & 9,1 & 9,6 & 14,1 & 7,3 & 10,0 & 10,7 & 10,1 \\
\hline 4 & 10,9 & 16,4 & 27,0 & 44,1 & 7,9 & 10,1 & 12,5 & 17,7 & 8,2 & 12,5 & 16,2 & 16,3 \\
\hline 5 & 10,1 & 14,3 & 25,9 & 44,6 & 7,9 & 9,1 & 11,1 & 16,2 & 9,4 & 11,2 & 15,3 & 16,3 \\
\hline 6 & 8,7 & 11,9 & 23,2 & 38,0 & 7,1 & 8,2 & 8,7 & 11,5 & 7,3 & 9,4 & 10,1 & 11,1 \\
\hline
\end{tabular}

Os resultados do teste de envelhecimento acelerado (Tabela 3), tanto o procedimento tradicional (96 horas), solução não saturada de $\mathrm{NaCl}$ (48h), quanto com solução saturada de $\mathrm{NaCl}$ (24 e 48 horas), permitiram a estratificação dos lotes em níveis de vigor, de maneira semelhante com os resultados obtidos na emergência de plântulas e índice de velocidade de emergência (Tabela 1).

$\mathrm{O}$ teste de envelhecimento acelerado tradicional estratificou os lotes conforme os níveis de vigor determinados pelos demais testes, somente no período de 96 horas de exposição, porém não foi eficiente devido à redução muito drástica da germinação. À medida que foi aumentando o tempo de exposição das sementes, a porcentagem de germinação foi decrescendo. Esse resultado também foi verificado em sementes de alface (BARBOSA et al., 2011) e salsa (TUNES et al., 2013). Segundo TUNES et al. (2011), esse efeito, provavelmente, deve-se ao alto teor de água atingido pelas sementes após o envelhecimento.
O período de envelhecimento de 48 horas utilizando solução salina saturada ou não possibilitaram o ranqueamento de lotes de sementes de coentro em três níveis de vigor, sem ocasionar reduções drásticas na porcentagem de plântulas normais ao final do teste.

A exposição das sementes ao envelhecimento acelerado com solução saturada de $\mathrm{NaCl}$ por 24 e 48 horas foi eficiente, selecionando-se o primeiro tratamento, porque, na separação de lotes de sementes de coentro em níveis de vigor, o menor período de execução é uma característica desejável nesse teste de vigor, fornecendo resultados de menor intervalo de tempo possível.

\section{CONCLUSÃo}

O teste de envelhecimento acelerado, utilizando solução salina saturada na combinação 24 horas a $41^{\circ} \mathrm{C}$, é adequado para avaliação do potencial

Tabela 3 - Germinação (\%) de lotes de sementes de coentro após quatro períodos de exposição ao teste de envelhecimento acelerado (EA), método tradicional, solução salina não saturada (SSNS), solução salina saturada (SSS). (Germination (\%) of coriander seed lots after four periods of exposure to the conventional accelerated aging, unsaturated salt solution and saturated salt solution tests). Capão do Leão, RS. 2013.

\begin{tabular}{|c|c|c|c|c|c|c|c|c|c|c|c|c|}
\hline \multirow[t]{2}{*}{ Lotes } & \multicolumn{4}{|c|}{---------Tradicional Tempo (horas)------- } & \multicolumn{4}{|c|}{--SSNS Tempo (horas)----------- } & \multicolumn{4}{|c|}{--SSS Tempo (horas) } \\
\hline & 24 & 48 & 72 & 96 & 24 & 48 & 72 & 96 & 24 & 48 & 72 & 96 \\
\hline 1 & $32 b$ & $19 b$ & $15 b$ & $09 \mathrm{~b}$ & $68 \mathrm{a}$ & $56 \mathrm{~b}$ & $58 \mathrm{a}$ & $40 \mathrm{a}$ & $63 b$ & $56 b$ & $59 b$ & $51 \mathrm{a}$ \\
\hline 2 & $70 \mathrm{a}$ & $58 \mathrm{a}$ & $44 a$ & $36 a$ & $73 a$ & $67 \mathrm{a}$ & $60 \mathrm{a}$ & $49 \mathrm{a}$ & $75 \mathrm{a}$ & $66 a$ & $66 \mathrm{a}$ & $55 \mathrm{a}$ \\
\hline 3 & $71 \mathrm{a}$ & $60 \mathrm{a}$ & $43 \mathrm{a}$ & $33 a$ & $70 \mathrm{a}$ & $63 a$ & $65 \mathrm{a}$ & $51 \mathrm{a}$ & $73 a$ & $69 a$ & $69 a$ & $51 \mathrm{a}$ \\
\hline 4 & $30 \mathrm{~b}$ & $16 \mathrm{~b}$ & $13 b$ & $01 \mathrm{c}$ & $65 \mathrm{~b}$ & $34 c$ & $51 b$ & $34 \mathrm{c}$ & $51 \mathrm{c}$ & $43 c$ & $55 \mathrm{~b}$ & $50 \mathrm{a}$ \\
\hline 5 & $70 a$ & $59 a$ & $42 a$ & $11 \mathrm{~b}$ & $52 \mathrm{~b}$ & $51 \mathrm{~b}$ & $28 \mathrm{c}$ & $16 \mathrm{~d}$ & $60 \mathrm{~b}$ & $55 \mathrm{~b}$ & $40 c$ & $36 \mathrm{~b}$ \\
\hline 6 & $73 a$ & $69 \mathrm{a}$ & $33 \mathrm{a}$ & $29 \mathrm{a}$ & $75 \mathrm{a}$ & $63 \mathrm{a}$ & $62 \mathrm{a}$ & $45 \mathrm{a}$ & $75 \mathrm{a}$ & $70 \mathrm{a}$ & $66 \mathrm{a}$ & $50 \mathrm{a}$ \\
\hline (DMS) & 10,5 & 14,6 & 14,5 & 7,5 & 8,5 & 6,3 & 7,2 & 7,8 & 6,0 & 7,0 & 5,5 & 9,5 \\
\hline $\mathrm{CV}(\%)$ & \multicolumn{4}{|c|}{18,15} & \multicolumn{4}{|c|}{11,23} & \multicolumn{4}{|c|}{14,07} \\
\hline
\end{tabular}

*Médias seguidas de mesma letra na coluna não diferem pelo teste de Tukey, em nível de probabilidade de $5 \%$.

Ciência Rural, v.46, n.1, jan, 2016. 
fisiológico de sementes de coentro. O período de 48 horas, tanto para o método de envelhecimento acelerado utilizando solução salina saturada ou solução salina não saturada, também é eficaz na avaliação da expressão do vigor em sementes de coentro.

\section{AGRADECIMENTOS}

Ao Conselho Nacional de Desenvolvimento Científico e Tecnológico (CNPq) e à Coordenação de Aperfeiçoamento de Pessoal de Nível Superior (CAPES), pelas bolsas concedidas.

\section{REFERÊNCIAS}

ÁVILA, P.V. et al. Teste de envelhecimento acelerado para avaliação do potencial fisiológico de sementes de rabanete. Revista Brasileira de Sementes, v.28, n.3, p.52-58, 2006. Disponível em: <http://www. scielo.br/pdf/rbs/v28n3/08.pdf>. Acesso em: 15 dez. 2013.

BRASIL, Ministério da Agricultura, Pecuária Abastecimento. Regras para análise de sementes. Secretaria de Defesa Agropecuária. Brasília: MAPA/ACS, 2009. 395p.

BHÉRING, M.C. et al. Teste de envelhecimento acelerado em sementes de pimenta. Revista Brasileira de Sementes, v.28, n.3, p.64-71, 2006. Disponível em: $<$ http://dx.doi.org/10.1590/S010131222006000300010>. Acesso em: 15 dez. 2013. doi: 10.1590/ S0101-31222006000300010.

BARBOSA, R.M. et al. Envelhecimento acelerado em sementes de alface. Ciência Rural, v.41, n.11, p.1899-1902, 2011. Disponível em: $<\mathrm{http}$ //dx.doi.org/10.1590/S0103-84782011005000138>. Acesso em: 15 dez. 2013. doi: 10.1590/S0103-84782011005000138.

COSTA, C.J. et al. Potencial fisiológico de sementes de brássicas com ênfase no teste de envelhecimento acelerado. Horticultura Brasileira, v.26, n.2, p.144-148, 2008. Disponível em: <http:// dx.doi.org/10.1590/S0102-05362008000200003>. Acesso em: 20 dez. 2013. doi: 10.1590/S0102-05362008000200003.

JIANHUA, Z.; McDONALD, M.B. The saturated salt accelerated aging test for small seeds crops. Seed Science and Techology, v.25, n.1, p.123-131, 1996.

MAGUIRE, J.D. Speed of germination aid in selection and evaluation for seedling and vigour. Crop Science, v.2, n.2, p.176-177, 1962.

MARCOS FILHO, J. Teste de envelhecimento acelerado. In: KRZYZANOWSKI, F.C. et al. (Eds.). Vigor de sementes: conceitos e testes. Londrina: ABRATES, 1999. p.3.1-3.24.
MARCOS FILHO, J. Relações água/semente. In: (Ed.). Fisiologia de sementes de plantas cultivadas. Piracicaba: FEALQ. 2005. p.169-196.

NAKAGAWA, J. Testes de vigor baseados no desempenho das plântulas. In: KRZYZANOWSKI, F. et al. (Eds.). Vigor de sementes: conceitos e testes. Londrina: ABRATES, 1999. Cap.2, p.2-24.

PEREIRA, M.F.S. et al. Qualidade fisiológica de sementes de coentro [Coriandrum sativum (L.)]. Revista Brasileira Plantas Medicinais, v.13, n. esp, p.518-522, 2011. Disponível em: <http://www.scielo.br/ pdf/rbpm/v13nspe/a02v13nspe.pdf $>$. Acesso em: 18 dez. 2013.

RAMOS, N.P. et al. Envelhecimento acelerado em sementes de rúcula (Eruca sativa L.). Revista Brasileira de Sementes, v.26, n.1, p.98-103, 2004. Disponível em: <http://dx.doi.org/10.1590/ S0101-31222004000100015>. Acesso em: 20 dez. 2013. doi: 10.1590/S0101-31222004000100015.

RODO, A.B. et al. Metodologia alternativa do teste de envelhecimento acelerado para sementes de cenoura. Scientia Agricola, v.57, n.2, p.289-292, 2000. Disponível em: <http:// dx.doi.org/10.1590/S0103-90162000000200015>. Acesso em: 18 dez. 2013. doi: 10.1590/S0103-90162000000200015.

TORRES, S.B.; BEZERRA-NETO, F. Teste de envelhecimento acelerado para avaliação do potencial fisiológico de sementes de urucum. Horticultura Brasileira, v.27, n.1, p.55-58, 2009 . Disponível em: <http://dx.doi.org/10.1590/S010205362009000100011>. Acesso em: 06 jan. 2014. doi: 10.1590/ S0102-05362009000100011.

TORRES, S.B. et al. Envelhecimento acelerado para avaliação do potencial fisiológico de sementes de melão. Horticultura Brasileira, v.27, n.1, p.70-75, 2009. Disponível em: <http:// dx.doi.org/10.1590/S0102-05362009000100014>. Acesso em: 20 dez. 2013. doi: 10.1590/S0102-05362009000100014.

TUNES, L.M. et al. Envelhecimento acelerado modificado para sementes de coentro (Coriandrum sativum L.) e sua correlação com outros testes de vigor. Revista Brasileira de Biociência, v.9, n.1, p.12-17, 2011. Disponível em: <http://www.ufrgs.br/seerbio/ojs/ index.php/rbb/article/view/1645/990>. Acesso em: 18 dez. 2013.

TUNES, L.M. et al. Envelhecimento acelerado em sementes de brócolis (Brassica oleracea L. var. italica Plenk). Bioscience Journal, v.28, n.2, p.173-179, 2012. Disponível em: <http:// www.seer.ufu.br/index.php/biosciencejournal/article/ view/11636/8475>. Acesso em: $18 \mathrm{dez} .2013$.

TUNES, L.M. et al. Accelerated aging to assess parsley seed vigor. Horticultura Brasileira, v.31, n.3, p.457-460, 2013. Disponível em: <http://dx.doi.org/10.1590/S010205362013000300018>. Acesso em: $18 \mathrm{dez}$. 2013. doi: 10.1590/ S0102-05362013000300018. 